\title{
Predictors of early versus late mortality in pelvic trauma patients
}

\author{
Hao Wang ${ }^{1 *}$, Richard D. Robinson ${ }^{1}$, Billy Moore², Alexander J. Kirk' ${ }^{1}$ Jessica Laureano Phillips², \\ Johnbosco Umejiego ${ }^{1}$, Joseph Chukwuma', Tyler Miller ${ }^{1}$, Donna Hassani ${ }^{3}$ and Nestor R. Zenarosa ${ }^{2}$
}

\begin{abstract}
Background: Risks of predicting time-related in-hospital mortality varies in pelvic trauma patients. We aim to identify potential independent risks predictive of time-related (early versus late) mortality among pelvic trauma patients.

Methods: Local trauma registry data from 2004 through 2013 were reviewed. Mortality causes and timing of death were investigated. Multivariate logistic regression identified independent risks predictive of early versus late mortality in pelvic trauma patients while adjusting for patient demographics (age, sex, race), clinical variables (initial vital signs, mental status, injury severity, associated injuries, comorbidities), and hospital outcomes (surgical interventions, crystalloid resuscitations, blood transfusions).

Results: We retrospectively collected data on 1566 pelvic trauma patients with a mortality rate of $9.96 \%(156 / 1566)$. Approximately $74 \%$ of patients died from massive hemorrhage within the first $24 \mathrm{~h}$ of hospitalization (early mortality). Revised trauma score (RTS), injury severity score (ISS), initial hemoglobin, direct transfer to operating room, and blood transfusion administration in the Emergency Department were considered independent risk factors predictive of early mortality. Age, ISS, and Glasgow Coma Scale (GCS) were deemed risk factors predictive of death after $24 \mathrm{~h}$ (late mortality).

Discussion: Given the fact of a substantial number of patients died within the first $24 \mathrm{~h}$ of hospital arrival, it is reasonable to consider the first $24 \mathrm{~h}$ of hospitalization as the appropriate window within which early mortality may be expected to occur in pelvic trauma patients. The risk factors associated with massive hemorrhage were strong predictors of early mortality, whereas late mortality predictors were more closely linked with comorbidities or in-hospital complications.
\end{abstract}

Conclusions: While risk factors predictive of early versus late mortality vary, ISS seems to predict both early and late mortality accurately in pelvic trauma patients.

Keywords: Pelvic trauma, Mortality, Time-related, Predictors

\section{Background}

Pelvic trauma is one of the most severe and complicated types of injury and is associated with relatively high mortality and morbidity [1-3]. Different independent risk factors predictive of in-hospital mortality in pelvic trauma have been reported. Results from a United Kingdom pelvic trauma registry study showed that patient age, initial blood pressure, mental status, injury severity score, and other associated injuries were independent risk factors for mortality [4]. Other international studies have not only confirmed these findings,

\footnotetext{
* Correspondence: HWang01@jpshealth.org

'Emergency Department, JPS Health Network, 1575 S. Main St, Fort Worth,

TX 76104, USA

Full list of author information is available at the end of the article
}

but have also affirmed other risk factors predictive of in-hospital mortality in pelvic trauma such as whether patients received packed red blood cell transfusions within the first $12 \mathrm{~h}(\mathrm{~h})$ of hospitalization and whether the patient was injured intentionally $[5,6]$. Several North American studies have asserted other in-hospital mortality predictors among pelvic trauma patients, such as low initial hemoglobin and direct transfer to the operating room (OR) [7-12]. Regardless of different risks predicting in-hospital mortality in pelvic trauma patients, the most common independent risk factor in the literature is severity of injuries as determined via revised trauma score (RTS) and injury severity score (ISS) systems $[13,14]$. 
In addition, literature also reported predictors vary with in-hospital mortality at different time points. Chong et al. [10] reported the majority of pelvic trauma patients whose deaths occurred within the first $72 \mathrm{~h}$ were due to pelvic hemorrhage, non-pelvic injury, or brain trauma, while those deaths occurring later $(>72 \mathrm{~h}$ ) were attributed to multisystem organ failure or acute respiratory distress syndrome (ARDS). Gunst et al. [15] reported different risks occurred in early $(\leq 4 \mathrm{~h})$ versus late $(>4 \mathrm{~h})$ mortality in pelvic trauma patients noting higher risk of late mortality in this group. Other evidence has also shown low hemoglobin and blood product administration are common early mortality predictors, whereas age is a common and consistent predictor of late mortality [12, 1618]. Severity of injuries scores including RTS and ISS, although valuable in predicting severity, do not effectively predict time-related mortality among pelvic trauma patients. Previous studies have shown that both RTS and ISS accurately identify severity of traumatic injuries and predict mortality in general [19-21]. Other tools have determined different values of predicting time-related mortality in pelvic trauma with either RTS or ISS [12, 22, 23]. Furthermore, though these studies reported the value of incorporating RTS and ISS in the prediction of early versus late hospital mortality their definitions of early mortality were different from other studies [19, 24]. Since in-hospital mortality predictors among pelvic trauma patients varies especially in the prediction of time-related mortality, it is important to differentiate early versus late in-hospital mortality, delineate its etiologies, and identify its appropriate predictors associated with time.

Generally, 'early' in-hospital mortality refers to patient death occurring within the first $24 \mathrm{~h}$ of hospital arrival while any death occurring beyond this point is considered 'late'. This is partly due to the diverse causes of death in pelvic trauma [7, 18]. Early in-hospital death among pelvic trauma patients may directly or indirectly occur because of massive hemorrhage, whereas surgical complications, infections, or multisystem organ failure may occur after $24 \mathrm{~h}$ and relates to 'late' in-hospital death [7, 18, 25-27]. Though different 'early' in-hospital mortalities were reported, it appears that no such study focused on the validation of defining 'early' versus 'late' hospital mortality. Given the fact that in-hospital complications may occur within the first 48 to $72 \mathrm{~h}$ of hospitalization $[11,28]$ and pelvic trauma patients hospitalized longer than $24 \mathrm{~h}$ may still have potential for significant active bleeding $[11,28,29,30]$, it is worthwhile to raise the question as to whether it is suitable to consider $24 \mathrm{~h}$ as a reliable discrimination point for early death in pelvic trauma patients. In addition, further identification and validation of factors capable of accurately predicting early versus late mortality in patients suffering pelvic trauma is necessary to increase physician awareness of patients' conditions and guide appropriate management.

Therefore, in order to better understand the causes of death and potential risks predictive of time-related mortality in pelvic trauma patients, we aimed to: 1) study what is a suitable window for early in-hospital mortality; 2) determine the common causes of early mortality; and 3) validate the different independent risk factors to predict early versus late mortality.

\section{Methods}

\section{Selection of participants}

Retrospective review of all patients entered into the local trauma registry during the period January 1, 2004 through December 31, 2013 was performed. The study included all adult (18 and over) patients with traumatic pelvic injuries that presented to the ED with signs of life (i.e., those not pronounced dead prior to or immediately on arrival to ED). Since this study focused on identifying independent risk factors associated with early versus late in-hospital mortality, patients missing final disposition data or for whom we were unable to identify survival status were excluded.

\section{Study design and protocol}

For the purpose of this study, mortality refers to all cause in-hospital mortality. We did not set out to identify the direct causes of death due to pelvic injuries; instead we focused on common causes of in-hospital mortality among pelvic trauma patients reported in the literature, including massive hemorrhage, other associated injuries, and severe in-hospital or trauma related complications. Because death due to massive hemorrhage rarely occurred beyond the first $72 \mathrm{~h}$ of hospital admission, for this study, we defined mortality due to massive hemorrhage as such if any one of the following criteria were met: 1) patient required more than an initial 10 units blood transfusion (either packed red blood cell or whole blood) and subsequently required additional blood transfusion until death or the $72 \mathrm{~h}$ end-point was reached; 2) persistent hypotension despite continuous blood transfusions with continued down-trending hemoglobin; or 3) immediate transfer to operating room (OR) upon arrival to the hospital to facilitate exploratory laparotomy and hemorrhage control to include packing procedure (intra-operative damagecontrol approach).

Mortality was determined to be due to other associated injuries if hemorrhage, which was associated with injuries apart from pelvic trauma, was controlled (e.g. intra-abdominal organ injury and significant intraabdominal bleeding was controlled) or the causes of massive hemorrhage were ruled out. In addition, patients meeting the following criteria were included in this group: 1) associated injuries confirmed by adjunct exams after hospital admission; 2) initial clinical 
presentation inconsistent with pelvic injuries; or 3) condition neither changed nor improved (e.g., mortality due to associated head injury was considered if patient did not meet hemorrhage criteria, initial presentation was altered mental status with confirmed intracranial pathology, and clinical condition worsened after hospital admission). Mortality classification could simultaneously encompass more than one associated injury if certain criteria were met. If a patient died before the completion of adjunct exams and treating physicians were unable to identify the associated injuries, an extensive chart review was conducted. If any of the following evidence (such as physician progress notes, consultant notes, imaging testing and lab results) were recorded in the patient's medical record indicating, but not limited to: infection, acute respiratory distress syndromes (ARDS), pulmonary embolism, single or multisystem organ failure, the cause of death was categorized as secondary to in-hospital or trauma related complication(s). Again, two or more causes of death could occur in one patient simultaneously.

Mortality was divided into four categories according to time, within the first $24,48,72$, or beyond $72 \mathrm{~h}$ of hospitalization. Causes of death were reviewed and determined independently by two physicians, each with ample trauma experience who are actively engaged in the management of trauma patients. If a discrepancy occurred, the study's Principle Investigator acted as the third reviewer rendering a final decision. Inter-observer agreement was measured.

Based on the results of mortality analysis, a discrimination point for early mortality was identified. Patients were then divided into two groups (early mortality [E group] and late mortality [L group]). Basic patient demographics (age, sex, race, mode of arrival) and ED clinical variables (initial vital signs, RTS, GCS, associated injuries, amount of crystalloid received, blood transfusion administration) were analyzed and compared. In order to determine the potential risk factors predictive of early versus late inhospital mortality, stepwise multivariate logistic regression analysis was performed. To determine whether early mortality in pelvic trauma patients could extend to $72 \mathrm{~h}$ post admission, dependent outcome variables $(24 \mathrm{~h}, 48 \mathrm{~h}$, and $72 \mathrm{~h}$ mortalities) were again used in multivariate logistic regression analysis. Their accuracy and discrimination levels were compared. The local institutional review board approved this study.

\section{Data analysis}

Student's $t$ Test was used to compare continuous variables between two groups, while Pearson Chi-square $(\chi 2)$ analysis was used to compare categorical variables. Inter-reader agreement testing was performed to determine the level of consistency among reviewers by using the kappa statistic ( $\kappa>0.4$, moderate agreement and $\kappa>0.6$, strong agreement). In order to identify independent risk factors predictive of early versus late mortality and to avoid potential confounders, clinical variables were entered into a stepwise multivariate logistic regression model. After preliminary data analysis, it was found that some variables had missing values for 0.4 to $6.2 \%$ of cases (Appendix). These missing data demonstrated an arbitrary pattern. Therefore, an iterative Markov Chain Monte Carlo (MCMC) method was used to impute the missing data in a multivariate normal model. Hosmer-Lemeshow test was performed to determine the goodness of fit for logistic regression model. C-statistic was used to assess the discriminatory power of the model. Models consisted of strong predictors if the $\mathrm{C}$-statistic value was greater than 0.8. Receiver operating characteristic (ROC) curves were drawn and the area under the ROC (AUC) measurements were derived to determine the accuracy of models in predicting early versus late mortality. All descriptive and statistical analyses were performed using Stata 12.0 (College Station, TX). A $p$ value less than 0.05 was considered statistically significant.

\section{Results}

A total of 1566 pelvic trauma patients met inclusion criteria. General patient information and clinical variables are reported in Table 1. Of those included, 156 died after admission, resulting in a total mortality rate of $9.96 \%$. Among all non-survivors, $57 \%$ died within the first $24 \mathrm{~h}$ of admission and $20 \%$ died after 7 days of hospitalization (Fig. 1). A total of 101 patients died within the first $72 \mathrm{~h}$ and 89 within the first $24 \mathrm{~h}$ of hospital arrival. Of those who died within the first $24 \mathrm{~h}, 74 \%(66 / 89)$ were due to massive hemorrhage (Table 2). A significantly low percentage of patient deaths were due to in-hospital or trauma related complications (Table 2). Investigator reviewed deaths due to hemorrhage and associated injuries resulted in strong inter-reader agreement scores $(\kappa=0.70, p<0.01$ and $\kappa=0.74, p<0.01$ ). The kappa coefficient of agreement on patient death due to inhospital or trauma related complication was 0.59 indicating a reasonable level of agreement $(p<0.01)$.

In order to determine independent risk factors predictive of in-hospital mortality, patients were divided into two groups. Patients who died within the first $24 \mathrm{~h}$ of arrival were placed in the 'early group' or E group, whereas those who died beyond $24 \mathrm{~h}$ were placed in the 'late group' or $\mathrm{L}$ group. Missing data were then imputed. The results of the Hosmer-Lemeshow goodness of fit test for logistic regression showed that the model fit the data well and the risk prediction was well calibrated $(p=0.34)$. Basic demographics and clinical information are listed in Table 3. L group tended to be older, have higher systolic blood pressure, heart rate, and hemoglobin, and received lower volume crystalloid and blood products resuscitation in the ED. Severity of injury, sex, race, mental status, associated 
Table 1 General Pelvic Trauma Patient Information

\begin{tabular}{|c|c|}
\hline \multicolumn{2}{|l|}{ Patient Demographics } \\
\hline Age (Mean Years, SD) & $42(18)$ \\
\hline Sex (Male, $n, \%)$ & $1015(65)$ \\
\hline \multicolumn{2}{|l|}{ Race } \\
\hline White $(n, \%)$ & $1008(64)$ \\
\hline African American $(n, \%)$ & $174(11)$ \\
\hline Hispanic ( $n, \%)$ & $293(19)$ \\
\hline Others $^{\mathrm{a}}(n, \%)$ & $91(6)$ \\
\hline \multicolumn{2}{|l|}{ ED Clinical Variables } \\
\hline Systolic BP (Mean mmHg, SD) & $122(31)$ \\
\hline Heart Rate (Mean, SD) & $94(24)$ \\
\hline Respiratory Rate (Mean, SD) & $18(5)$ \\
\hline GCS (Mean, SD) & $12.9(4.0)$ \\
\hline \multicolumn{2}{|l|}{ RTS } \\
\hline (Mean, SD) & $7.15(1.54)$ \\
\hline (Median, IQR) & $7.84(0)$ \\
\hline \multicolumn{2}{|l|}{ ISS } \\
\hline (Mean, SD) & $20(13)$ \\
\hline (Median, IQR) & $17(18)$ \\
\hline Crystalloid Received at ED (Mean ml, SD) & $1836(1463)$ \\
\hline \multicolumn{2}{|l|}{ Type of Injury } \\
\hline Blunt $(n, \%)$ & $1520(97)$ \\
\hline Penetrating $(n, \%)$ & $46(3)$ \\
\hline \multicolumn{2}{|l|}{ Type of pelvic injury } \\
\hline Ilium $(n, \%)$ & $234(15)$ \\
\hline Ischium (n, \%) & $61(4)$ \\
\hline Pubis $(n, \%)$ & $702(45)$ \\
\hline Sacrum/coccyx (n, \%) & $429(27)$ \\
\hline Acetabulum ( $n, \%)$ & $715(46)$ \\
\hline Unclear $(n, \%)$ & $109(7)$ \\
\hline Associated Injuries $(n, \%)^{\mathrm{b}}$ & $734(47)$ \\
\hline Head $(n, \%)$ & $339(22)$ \\
\hline Chest $(n, \%)$ & $215(14)$ \\
\hline Abdomen ( $n, \%)$ & $281(18)$ \\
\hline Extremity $(n, \%)$ & $313(20)$ \\
\hline Blood Transfusion at ED (yes, \%) & $166(11)$ \\
\hline Hgb (Mean g, SD) & $12.8(2.4)$ \\
\hline Transfer Directly to OR (yes, \%) & $275(18)$ \\
\hline
\end{tabular}

Abbreviations: SD standard deviation, BP blood pressure, GCS Glasgow Coma Scale, $R T S$ revised trauma score, IQR interquartile range, ISS Injury Severity Score, ED Emergency Department, $\mathrm{Hg} b$ hemoglobin, OR operating room ${ }^{a}$ : others include Asian, Pacific Islander, and Native American. ${ }^{b}$ : Associated injuries: injuries other than these four regions are not reported in this study

injuries, and whether emergent surgical intervention was required, showed no statistically significant difference between groups (Table 3 ). Early versus late mortality predictive risk factors are reported along with their C-statistics

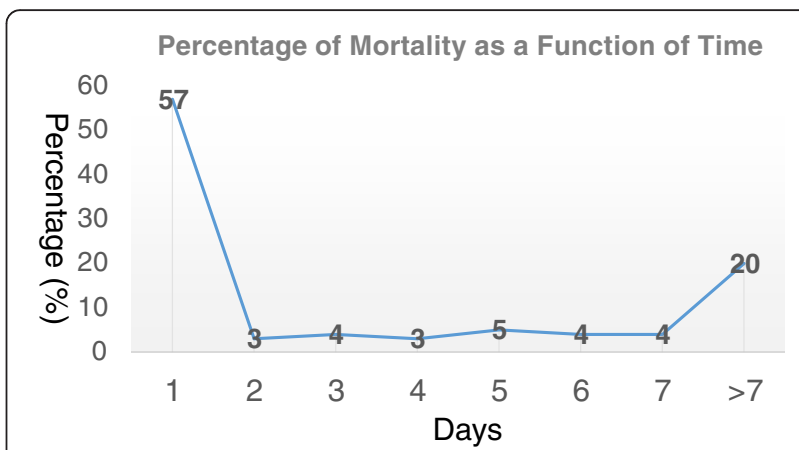

Fig. 1 Percentage of Mortality as a Function of Time among All Deceased Pelvic Trauma Patients

(Table 4). ROC curves of models predicting early and late mortality are shown in Fig. 2. We found no statistically significant difference in independent risk factors among patients who died within 24, 48, or $72 \mathrm{~h}$ of hospital arrival. The C-statistics in three models predicting early mortality within 24, 48, and $72 \mathrm{~h}$ of hospitalization were 0.9505 , 0.9501 , and 0.9493 respectively $(p>0.05)$.

\section{Discussion}

High mortality rates occur among patients with severe trauma, especially among those with pelvic injuries [1]. Previous studies have reported increased early mortality rates in pelvic trauma patients primarily due to massive hemorrhage and severity of traumatic injuries. Other studies have reported that late mortality among patients with pelvic injuries may be due to surgical complication, infection, and/or multisystem organ failure [4, 10, 31]. Given the fact that, in this study, a substantial number of patients died within the first $24 \mathrm{~h}$ of hospital arrival and the majority of them were due to hemorrhage, it might be appropriate to consider the first $24 \mathrm{~h}$ as a suitable discrimination point for early mortality. Risk factors predictive of early mortality occurred in pelvic trauma patients with less accuracy beyond the first $24 \mathrm{~h}$ [19;23]. Independent risk factors varied in the prediction of early versus late mortalities. The risk factors associated with massive hemorrhage were strong predictors of early mortality, whereas other factors were more closely linked with comorbidities or in-hospital complications. While severity of injury can predict in-

Table 2 Causes of Mortality at Specific Time Intervals in Pelvic Trauma Patients

\begin{tabular}{llll}
\hline Hospital Arrival & Hemorrhage & Associated Injuries & Complications $^{a}$ \\
\hline $0-24 \mathrm{~h}$ & $66(74 \%)$ & $43(48 \%)$ & $3(3 \%)$ \\
$24-48 \mathrm{~h}$ & $3(60 \%)$ & $4(80 \%)$ & $0(0)$ \\
$48-72 \mathrm{~h}$ & $6(86 \%)$ & $3(43 \%)$ & $1(14 \%)$ \\
$>72 \mathrm{~h}$ & $1(2 \%)$ & $35(64 \%)$ & $48(87 \%)$ \\
\hline
\end{tabular}

0-24 h: including $24 \mathrm{~h} ; 24-48 \mathrm{~h}$ : including $48 \mathrm{~h}$, not including $24 \mathrm{~h} ; 48-72 \mathrm{~h}$ : including $72 \mathrm{~h}$, not including $48 \mathrm{~h}$; ${ }^{\mathrm{a}}$ complications refer to in-hospital or trauma related complications as addressed in detail in Methods section 
Table 3 Comparisons of General Information in Pelvic Trauma Patients: Early versus Late Mortality

\begin{tabular}{|c|c|c|c|}
\hline & E group $(n=89)$ & L group $(n=67)$ & $P$ value \\
\hline \multicolumn{4}{|l|}{ Patient Demographics } \\
\hline Age - year, mean (SD) & $44(18)$ & $51(21)$ & $<0.05$ \\
\hline Gender —Male, yes (\%) & 78 & 70 & 0.30 \\
\hline \multicolumn{4}{|l|}{ Race } \\
\hline White (\%) & 68 & 75 & \multirow[t]{4}{*}{$<0.05$} \\
\hline African-American (\%) & 17 & 6 & \\
\hline Hispanic (\%) & 16 & 12 & \\
\hline Others $^{\mathrm{a}}(\%)$ & 0 & 7 & \\
\hline \multicolumn{4}{|l|}{ Clinical Variables upon Patient Arrival at ED } \\
\hline Systolic BP —mmHg, mean (SD) & $74(50)$ & $107(37)$ & $<0.01$ \\
\hline Heart Rate — bpm, mean (SD) & $87(52)$ & $102(34)$ & 0.05 \\
\hline Respiratory Rate — rpm, mean (SD) & $13(10)$ & $15(6)$ & 0.12 \\
\hline GCS - number, mean (SD) & $6.67(4.96)$ & $7.95(5.27)$ & 0.13 \\
\hline \multicolumn{4}{|l|}{ RTS } \\
\hline (Mean, SD) & $3.93(2.84)$ & $5.41(2.09)$ & $<0.01$ \\
\hline (Median, IQR) & $4.09(5.22)$ & $5.97(3.75)$ & 0.12 \\
\hline \multicolumn{4}{|l|}{ ISS } \\
\hline (Mean, SD) & $37(14)$ & $36(14)$ & 0.63 \\
\hline (Median, IQR) & $34(18)$ & $34(14)$ & 1.00 \\
\hline Crystalloid Received at ED —ml, mean (SD) & $2678(1790)$ & $2502(2114)$ & 0.61 \\
\hline \multicolumn{4}{|l|}{ Type of injury } \\
\hline Blunt (\%) & 94 & 97 & \multirow[t]{2}{*}{0.43} \\
\hline Penetrating (\%) & 6 & 2 & \\
\hline \multicolumn{4}{|l|}{ Location of pelvic injury } \\
\hline Ilium —n, (\%) & $12(18)$ & $9(15)$ & 0.60 \\
\hline Ischium-n, (\%) & $2(3)$ & $3(5)$ & 0.59 \\
\hline Pubis — n, (\%) & $40(59)$ & $36(58)$ & 0.93 \\
\hline Sacrum/coccyx 一n, (\%) & $19(29)$ & $13(21)$ & 0.31 \\
\hline Acetabulum —n, (\%) & $28(41)$ & $26(42)$ & 0.93 \\
\hline Unclear $-n_{,}(\%)$ & $22(25)$ & $5(7)$ & $<0.01$ \\
\hline Associated Injuries — $n$, (\%) & $88(99)$ & $65(97)$ & 0.40 \\
\hline Head $-n,(\%)$ & $43(48)$ & $40(60)$ & 0.16 \\
\hline Chest —n, (\%) & $55(62)$ & $40(60)$ & 0.79 \\
\hline Abdomen $-n,(\%)$ & $51(57)$ & $37(55)$ & 0.80 \\
\hline Extremities — n, (\%) & $57(65)$ & $39(59)$ & 0.47 \\
\hline Blood transfusion at ED (yes, \%) & 52 & 34 & $<0.05$ \\
\hline $\mathrm{Hgb} — \mathrm{~g}$, mean (SD) & $9.7(2.7)$ & $11.9(4.4)$ & $<0.01$ \\
\hline History of Cardiovascular Diseases (yes, \%) & 11 & 13 & 0.67 \\
\hline Transfer Directly to OR (yes, \%) & 49 & 39 & 0.19 \\
\hline
\end{tabular}

Abbreviations: $E$ group refers to pelvic trauma patients experiencing early death, $L$ group refers to pelvic trauma patients experiencing late death, $S D$ standard deviation, BP blood pressure, bpm beats per minute, rpm respirations per minute, GCS Glasgow Coma Scale, RTS revised trauma score, IQR interquartile range, ISS Injury Severity Score, ED Emergency Department, Hgb hemoglobin, OR operating room

a percentages may not add up to $100 \%$ due to rounding up. Others includes Asian, Pacific Islander, and Native American 
Table 4 Independent Risk Factors Predictive of Early versus Late Mortality in Pelvic Trauma Patients using Multivariate Logistic Regression Analysis

Adjusted Odds Ratios (95\% Cl) C-statistics

\begin{tabular}{lll}
\hline Early Mortality & \\
Blood Transfusion at ED & $2.14(1.19-3.86)$ & 0.72 \\
Transfer to OR from ED & $2.15(1.21-3.81)$ & 0.67 \\
ISS & $1.04(1.02-1.06)$ & 0.85 \\
RTS & $0.60(0.53-0.69)$ & 0.87 \\
Hgb & $0.73(0.66-0.82)$ & 0.82 \\
Final Model & & 0.95 \\
Late Mortality & $1.05(1.03-1.06)$ & \\
Age & $1.08(1.05-1.11)$ & 0.63 \\
ISS & $0.84(0.78-0.89)$ & 0.85 \\
GCS & & 0.81 \\
Final Model & & 0.89 \\
\hline
\end{tabular}

Abbreviations: $\mathrm{Cl}$ confidence interval, $O R$ operating room, ISS injury severity score, RTS revised trauma score, Hgb hemoglobin, GCS Glasgow Coma Scale

hospital mortality, it appears in this study only ISS accurately predicts both early and late mortality in pelvic trauma patients. Our findings are consistent with the majority of previous studies focusing on pelvic trauma but with the added emphasis on identifying time-related mortality and its predictive risk factors $[4,7,12]$. Identifying potential time-related mortality risk factors will alert physicians to take appropriate early actions thus improving outcomes in pelvic trauma patients.

Previous studies identified early death with similar causes extending up to $72 \mathrm{~h}$ post admission $[5,10]$. Very few studies, however, reported the percentage of early deaths separately at $24 \mathrm{~h}$ increments along with causes of death [11]. Those who did report time-related mortality showed the majority of early deaths occurred in the first $24 \mathrm{~h}$ of hospital admission and their causes of death were mostly associated with massive hemorrhage $[7,16]$. Our study demonstrates similar findings while also comparing patients who died within 24,48 , and $72 \mathrm{~h}$ of hospital admission. Though a small percentage of patients died beyond $24 \mathrm{~h}$ from massive bleeding, it did not affect the accuracy of early mortality prediction in our study.

Mortality prediction without consideration of the timeline in which it occurred may not provide accurate results among pelvic trauma patients. When prediction is narrowed to either early or late mortality, less variety occurs. Our findings are consistent with the literature suggesting that risks associated with bleeding, such as initial hemoglobin, initial blood product administration, and severity of injury, are reliable independent risk factors predictive of early mortality, while age and associated injuries are reliable predictors of late mortality [4, 12, 28]. Some studies found that severe head injuries are predictors of early death while other studies suggest that patients with severe head injuries tended to die after $24 \mathrm{~h}$ of hospitalization $[5,10,23,32]$. Our findings showed no significant difference in terms of mortality associated with these injuries. RTS was shown to predict early mortality whereas GCS predicted late mortality alone. This indicates that GCS should heavily weigh in on late mortality prediction in pelvic trauma patients. We are not certain, however, whether low GCS is directly linked to severity of head injuries in this study as this was not specifically investigated.

Both RTS and ISS are predictive of early mortality, though only ISS is predictive of late mortality. RTS is calculated based on patient GCS, blood pressure, and respiratory rate, which are closely related to hemodynamic stability. We therefore conclude this to be an accurate predictor of early mortality $[14,33]$. ISS is determined by associated injuries and is calculated utilizing an abbreviated injury scale in each of the 6 body regions which summarizes the patient's general condition in addition to the severity of the injuries within each region [34]. ISS not only identifies current injury severity but also estimates severity of associated injuries in pelvic trauma patients. As there is
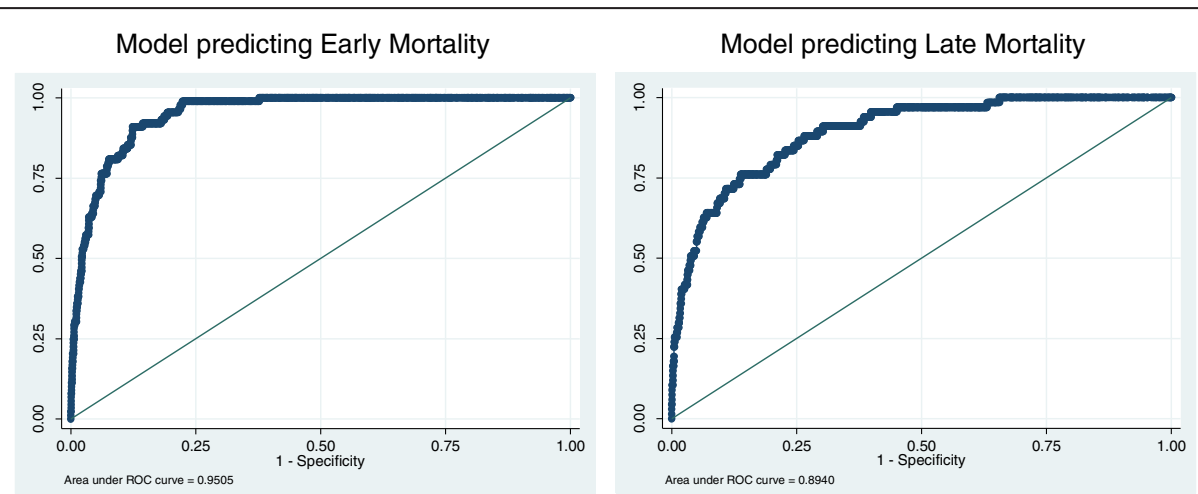

Fig. 2 ROC Curves of Models Predicting Early and Late Mortality in Pelvic Trauma Patients 
often a high rate of associated complex injuries in pelvic trauma, ISS becomes a reasonable variable to predict mortality [23, 35]. Our study validated the incorporation of ISS in predicting both early and late mortality. With the popular use of electronic medical documentation systems, it is now practical to calculate ISS by computer thereby providing real-time guidance to emergency physicians and trauma surgeons with respect to patient dispositions.

\section{Limitations}

Retrospective study designs cannot demonstrate causality due to limited information accuracy, missing data and potential selection bias. The direct cause of death cannot be determined precisely without performing an autopsy in each patient and physician judgement, while determining cause of death, might lead to subjective bias. It is, however, unrealistic to autopsy every study patient to more accurately determine the cause of death. Therefore, all-cause in-hospital mortality was the outcome measurement in this study. Furthermore, two physicians conducted intensive chart reviews independently. This approach reduced the risk of bias. We were unable to include all clinical variables for mortality analysis such as in-hospital medical or surgical complications, patient baseline comorbidities, or prehospital transportation times, which could render predictions less accurate. Future prospective studies focusing on the validation of these risk factors as predictive of early and late mortality in pelvic trauma patients are warranted.

\section{Conclusions}

It is reasonable to consider the first $24 \mathrm{~h}$ of hospitalization as the appropriate window within which early mortality

Table 5 Missing Data Rate of Variables used in this Study

\begin{tabular}{lll}
\hline Variables & Missing Data $(N)$ & Missing Rate (\%) \\
\hline Age & 0 & 0 \\
Gender & 0 & 0 \\
Race & 0 & 0 \\
Mechanisms of Injury & 0 & 0 \\
(Blunt/Penetrating) & \\
Receiving Blood Transfusion at ED & 0 & 0 \\
Transfer to Operating Room & 0 & 0 \\
Injury Severity Score & 6 & 0.4 \\
ED Pulse & 21 & 1.3 \\
ED Systolic Blood Pressure & 24 & 1.5 \\
ED Glasgow Coma Scale & 60 & 3.8 \\
ED Respiratory Rate & 65 & 4.2 \\
Hemoglobin & 97 & 6.2 \\
Revised Trauma Score & 97 & 6.2 \\
\hline
\end{tabular}

Abbreviation: N number, ED Emergency Department may be expected to occur in pelvic trauma patients. The risks predictive of early versus late mortality are different. Patient initial hemoglobin level, patient RTS and ISS, emergent blood transfusion, and emergent surgical intervention are independent risk factors for early mortality. Patient age, ISS, and mental status on arrival are independent risk factors for late mortality. Overall, ISS seems to predict both early and late mortality well in pelvic trauma patients.

\section{Appendix}

\section{Competing interests}

The authors declare that they have no competing interests.

\section{Authors' contributions}

$H W, R D R$, and NRZ conceived the study and developed the design in consultation with all of the authors. AJK, JLP, JU, JC, TM, and DH assembled the dataset and collected the data. HW, BM, JLP and JU conducted the statistical analyses. HW, RDR, JLP, and NRZ drafted the articles. All authors read and approved the final manuscript. HW takes responsibility for the paper as a whole.

\section{Author details}

${ }^{1}$ Emergency Department, JPS Health Network, 1575 S. Main St, Fort Worth, TX 76104, USA. ${ }^{2}$ Research Institute, JPS Health Network, 1500 S. Main St, Fort Worth, TX 76104, USA. ${ }^{3}$ University of South Florida, Morsani College of Medicine, 12901 Bruce B. Downs Blvd., MDC061, Tampa, FL 33612-4799, USA.

Received: 9 September 2015 Accepted: 4 March 2016

Published online: 10 March 2016

\section{References}

1. Prieto-Alhambra D, Aviles FF, Judge A, et al. Burden of pelvis fracture: a population-based study of incidence, hospitalisation and mortality. Osteoporos Int. 2012:23:2797-803.

2. Cannada LK, Taylor RM, Reddix R, et al. The Jones-Powell Classification of open pelvic fractures: a multicenter study evaluating mortality rates. J Trauma Acute Care Surg. 2013;74:901-6.

3. Alonso JE, Lee J, Burgess AR, et al. The management of complex orthopedic injuries. Surg Clin North Am. 1996;76:879-903.

4. Giannoudis PV, Grotz MR, Tzioupis C, et al. Prevalence of pelvic fractures, associated injuries, and mortality: the United Kingdom perspective. J Trauma. 2007;63:875-83.

5. Holstein $\mathrm{JH}$, Culemann U, Pohlemann T. What are predictors of mortality in patients with pelvic fractures? Clin Orthop Relat Res. 2012;470:2090-7.

6. Gabbe BJ, de Steiger R, Esser M, et al. Predictors of mortality following severe pelvic ring fracture: results of a population-based study. Injury. 2011; 42:985-91.

7. Smith W, Williams A, Agudelo J, et al. Early predictors of mortality in hemodynamically unstable pelvis fractures. J Orthop Trauma. 2007:21:31-7.

8. Bramos A, Velmahos GC, Butt UM, et al. Predictors of bleeding from stable pelvic fractures. Arch Surg. 2011;146:407-11.

9. Starr AJ, Griffin DR, Reinert CM, et al. Pelvic ring disruptions: prediction of associated injuries, transfusion requirement, pelvic arteriography, complications, and mortality. J Orthop Trauma. 2002;16:553-61.

10. Chong $\mathrm{KH}$, DeCoster T, Osler T, et al. Pelvic fractures and mortality. Iowa Orthop J. 1997:17:110-4.

11. Abdelrahman $\mathrm{H}$, El-Menyar A, Al-Thani $\mathrm{H}$, et al. Time-based trauma-related mortality patterns in a newly created trauma system. World J Surg. 2014;38: 2804-12.

12. Ali J, Ahmadi KA, Williams JI. Predictors of laparotomy and mortality in polytrauma patients with pelvic fractures. Can J Surg. 2009;52:271-6. 
13. Torok A, Bancu S, Neagoe R, et al. The utility of the predictive scores in polytrauma with abdomino-pelvic injuries: a series of 38 patients. Chirurgia (Bucur). 2014;109:44-7.

14. Watts HF, Kerem Y, Kulstad EB. Evaluation of the revised trauma and injury severity scores in elderly trauma patients. J Emerg Trauma Shock. 2012;5: $131-4$.

15. Gunst M, Ghaemmaghami V, Gruszecki A, et al. Changing epidemiology of trauma deaths leads to a bimodal distribution. Proc (Bayl Univ Med Cent). 2010;23:349-54

16. Pohlemann $T$, Stengel $D$, Tosounidis $G$, et al. Survival trends and predictors of mortality in severe pelvic trauma: estimates from the German Pelvic Trauma Registry Initiative. Injury. 2011;42:997-1002.

17. Haddad SH, Yousef ZM, Al-Azzam SS, et al. Profile, outcome and predictors of mortality of abdomino-pelvic trauma patients in a tertiary intensive care unit in Saudi Arabia. Injury. 2015:46:94-9.

18. Arroyo W, Nelson KJ, Belmont Jr PJ, et al. Pelvic trauma: What are the predictors of mortality and cardiac, venous thrombo-embolic and infectious complications following injury? Injury. 2013;44:1745-9.

19. Dong JL, Zhou DS. Management and outcome of open pelvic fractures: a retrospective study of 41 cases. Injury. 2011;42:1003-7.

20. O'Sullivan RE, White TO, Keating JF. Major pelvic fractures: identification of patients at high risk. J Bone Joint Surg Br. 2005;87:530-3.

21. Banerjee S, Barry MJ, Paterson JM. Paediatric pelvic fractures: 10 years experience in a trauma centre. Injury. 2009:40:410-3.

22. Gustavo PJ, Coimbra R, Rasslan S, et al. The role of associated injuries on outcome of blunt trauma patients sustaining pelvic fractures. Injury. 2000;31:677-82

23. Lunsjo K, Tadros A, Hauggaard A, et al. Associated injuries and not fracture instability predict mortality in pelvic fractures: a prospective study of 100 patients. J Trauma. 2007;62:687-91.

24. Dente CJ, Feliciano DV, Rozycki GS, et al. The outcome of open pelvic fractures in the modern era. Am J Surg. 2005;190:830-5.

25. Guthrie HC, Owens RW, Bircher MD. Fractures of the pelvis. J Bone Joint Surg Br. 2010;92:1481-8.

26. Giannoudis PV, Pape HC. Damage control orthopaedics in unstable pelvic ring injuries. Injury. 2004;35:671-7.

27. Burkhardt M, Kristen A, Culemann U, et al. Pelvic fracture in multiple trauma: are we still up-to-date with massive fluid resuscitation? Injury. 2014;45 Suppl 3:S70-5.

28. Kataoka Y, Minehara H, Shimada K, et al. Sepsis caused by peripelvic soft tissue infections in critically injured patients with multiple injuries and unstable pelvic fracture. J Trauma. 2009;66:1548-54

29. Tang CH, Shivji F, Forward D. Major haemorrhage in pubic rami fractures. BMJ Case Rep. 2015;2015:bcr2014208088. doi:10.1136/bcr-2014-208088.

30. Sandri A, Regis D, Bizzotto N. Delayed bleeding and pelvic haematoma after low-energy osteoporotic pubic rami fracture in a warfarin patient: an unusual cause of abdominal pain. Case Rep Emerg Med. 2014;2014:783268.

31. Papakostidis C, Giannoudis PV. Pelvic ring injuries with haemodynamic instability: efficacy of pelvic packing, a systematic review. Injury. 2009:40 Suppl 4:S53-61.

32. Ooi CK, Goh HK, Tay SY, et al. Patients with pelvic fracture: what factors are associated with mortality? Int J Emerg Med. 2010;3:299-304

33. Guzzo JL, Bochicchio GV, Napolitano LM, et al. Prediction of outcomes in trauma: anatomic or physiologic parameters? J Am Coll Surg. 2005;201:891-7.

34. Copes WS, Champion HR, Sacco WJ, et al. The Injury Severity Score revisited. J Trauma. 1988;28:69-77.

35. Akhavan AG, Mohammadian A. Comparison of the RTS and ISS scores on prediction of survival chances in multiple trauma patients. Acta Chir Orthop Traumatol Cech. 2012:79:535-9.

\section{Submit your next manuscript to BioMed Central and we will help you at every step:}

- We accept pre-submission inquiries

- Our selector tool helps you to find the most relevant journal

- We provide round the clock customer support

- Convenient online submission

- Thorough peer review

- Inclusion in PubMed and all major indexing services

- Maximum visibility for your research

Submit your manuscript at www.biomedcentral.com/submit
Biomed Central 